\title{
Evolutionary Marginal Cost Pricing Scheme Implementation Based on Stochastic Traffic Flow Information
}

\author{
Wei $\mathrm{Xu}^{1}$ and Agachai Sumalee ${ }^{2}$ \\ ${ }^{1}$ School of Management and Engineering, Nanjing University, Nanjing 210093, China \\ ${ }^{2}$ Department of Civil Engineering, Faculty of Engineering, King Mongkut's Institute of Technology Ladkrabang, \\ Bangkok 10520, Thailand \\ Correspondence should be addressed to Wei Xu; xuwei@nju.edu.cn
}

Received 16 July 2014; Accepted 6 October 2014

Academic Editor: Ricardo Aguilar-López

Copyright (C) 2015 W. Xu and A. Sumalee. This is an open access article distributed under the Creative Commons Attribution License, which permits unrestricted use, distribution, and reproduction in any medium, provided the original work is properly cited.

\begin{abstract}
Traditionally, to implement the first-best marginal cost pricing scheme in a traffic network requires the information on the exact demand function or true origin-destination demand, which, however, is rarely available in practice. To overcome this dilemma, the trial-and-error method has been proposed to find the marginal cost toll through an iterative process using the observed traffic volumes. This method guarantees the convergence of tolls and flows to the system optimal state under the assumption of deterministic traffic conditions. However, in reality, the uncertainty of transportation network has been recognized well that induces the variability of link flow and travel time. Therefore, this paper proposes an evolutionary implementation method that iteratively finds the first-best marginal cost toll pattern according to the observed stochastic link flow information and the known travel time functions. The proof of the convergence of the iterative algorithm is presented. The paper also analyzes the effect of the sampling error of the link flow data on the convergence of the algorithm and shows that the biases from the flow observation will not affect the convergence. The numerical tests are provided for the illustration of the algorithm.
\end{abstract}

\section{Introduction}

The fundamental principle of road pricing, based on the concept of marginal social cost, is to impose the tolls equivalent to the externality incurred by road users to realize the socially optimal flow pattern. For a single road, the optimal toll is shown to be equal to $v \cdot d t(v) / d v$, where $v$ is the link flow and $d t(v) / d v$ is the derivative of the link travel time function. This principle is also proven to be applicable to the network case in which it is often referred to as the "first-best" road pricing. To implement this ideal pricing scheme in a traffic network, the information on the exact demand function or true origindestination (O-D) demand must be obtained. However, this is rarely available in practice in which most of the information on the demand function and O-D demand is often based on statistical estimations. In practice, on the other hand, the traffic volume data are observable and available in most cities. Li $[1,2]$ verified that the road pricing could go ahead on a trial-and-error basis without demand functions, which was first conjectured by Vickrey [3] and Downs [4]. The toll updating method developed by $\mathrm{Li}$ [2] is just for a single road link, and it was soon extended to a general network by Yang et al. [5], which proposed an efficient trial-and-error method to find the first-best pricing through an iterative process using the observed traffic volumes. The approach can guarantee the convergence of the tolls and flows to the system optimal state and successfully avoid the difficulty to acquire demand information. However, it is only proved under the assumption of deterministic traffic environment. In reality, the transportation network contains lots of stochastic factors including weather conditions, traffic incidents, and special events, which contribute to roadway capacity variation and travel demand fluctuation from day to day.

Recently, substantial studies have focused on the uncertainty of transportation network. An uncertain traffic environment implies that link flows and travel times are stochastic, and thereby the reliability of route travel time plays an important role in traveler's route choice behavior. Various 
reliability-based traffic assignment models were developed in view of the day-to-day demand fluctuations $[6,7]$ and link capacity variations [8]. A good understanding of the uncertainty is pretty important not only in traffic assignment problem, but also in network design problem (NDP). Patil and Ukkusuri [9] showed that neglecting the network uncertainty would lead to a suboptimal design. Several models that tackle the uncertainty issue in NDPs have been proposed recently in the literature ([10-14], etc.). Chen et al. [15] gave a complete literature review on NDPs with uncertainty. The effects of uncertain demand and supply have also been recognized in a variety of road pricing and tradable credit schemes design ([16-19], etc.). However, few studies pay attention to the question whether one can use the stochastic flow information collected in practice to define the marginal cost toll on a stochastic network.

This paper tackles the above research question in which a trial-and-error method is again proposed to iteratively calculate the first-best marginal-cost toll based on the observable stochastic link flows. This paper postulates that the network uncertainty is caused by the stochastic day-to-day travel demand (e.g., $[6,7,20]$ ). Hence the link traffic flow and link travel time are both stochastic too. Under this stochastic network ( $\mathrm{SN}$ ), the travelers' route choice decisions are assumed to follow the user equilibrium (UE) principle aiming to minimize their expected travel time. On the other hand, the system optimum (SO) is assumed to minimize the expected total travel time (for the fixed demand case). In order to distinguish them from those under the deterministic network, in this paper, they are referred to as SN-UE and SN-SO, respectively. The paper investigates the relationship between the SN-UE and SN-SO to establish the first-best marginal cost pricing for $\mathrm{SN}$ (SN-MCP), which is different from the original MCP in deterministic network. In other words, we cannot obtain the SN-MCP by using the expected values to do substitution. In the newly proposed trial-anderror algorithm, each trial toll is determined by the SN-MCP expression. That algorithm only requires the information on the statistics of the observed link flows and travel time functions. The distribution parameters of stochastic O-D demand are not needed. The paper proves the convergence of the iterative algorithm and also analyzes the effect of the sampling error of the link flow data on the convergence of the algorithm, in which the observation period may not be sufficiently long to deduce the true mean and variance of the link flow. We show that the biases from the flow observation will not affect the convergence of the optimal toll and flow pattern.

The outline of the paper is as follows. First, we derive the general formulation of the SN-MCP and show a closed-form formulation/calculation of the SN-MCP for a special case. Then, the trial-and-error algorithm for evolutionary implementation of the marginal cost pricing based on stochastic link traffic volumes is developed. The convergence properties are analyzed and proved. Following this, two numerical examples are examined to demonstrate the validity of the proposed method and exhibit the difference of the SN-MCP and original MCP. Finally, some conclusions are given.

\section{Marginal Cost Pricing under Stochastic Network}

The MCP principle states that travelers using congested roads should pay a toll equal to the difference between the marginal social and private cost in order to minimize the total system cost if demands are fixed or maximize the social welfare if demands are variable. In previous literatures, when all link flows, and thereby all link travel times, are deterministic, the MCP is expressed as follows:

$$
\mathrm{MCP}=\frac{\partial\left[\sum_{a \in A} v_{a} t_{a}\left(v_{a}\right)\right]}{\partial v_{a}}-t_{a}\left(v_{a}\right)=v_{a} \cdot \frac{d t_{a}\left(v_{a}\right)}{d v_{a}},
$$

where $v_{a}$ is the amount of traffic flow on link $a, t_{a}\left(v_{a}\right)$ is the private cost for traversing link $a$ and the derivative of the total system cost, $\sum_{a \in A} v_{a} t_{a}\left(v_{a}\right)$, with respect to the fact that $v_{a}$ is the marginal social cost.

Now, in this paper, travel demand uncertainty is taken into account. The stochastic demands give rise to stochastic link flows and stochastic link travel times. In this situation, it is obvious that we need to modify the expression of the MCP in (1) to make it well defined. If using capital $V_{a}$ to represent the stochastic link flow, one argument is that we can simply substitute the expected value of $V_{a}$ (i.e., $\left.E\left[V_{a}\right]\right)$ into the original MCP, $v_{a} \cdot d t_{a}\left(v_{a}\right) / d v_{a}$. In this way, the expression of the original MCP changes to $E\left[V_{a}\right] \cdot d t_{a}\left(E\left[V_{a}\right]\right) / d E\left[V_{a}\right]$. However, noting that $t_{a}\left(E\left[V_{a}\right]\right)$ is not equal to $E\left[t_{a}\left(V_{a}\right)\right]$ (the mean link travel time) with any nonlinear link travel time function, such a substitution generally cannot provide us the real difference between the marginal social and private cost. In the following analysis, it will be further seen that even if we take $E\left[t_{a}\left(V_{a}\right)\right]$ to replace $t_{a}\left(v_{a}\right)$ rather than $t_{a}\left(E\left[V_{a}\right]\right)$, that is, the original MCP changes to $E\left[V_{a}\right] \cdot d E\left[t_{a}\left(V_{a}\right)\right] / d E\left[V_{a}\right]$, it is not the real MCP under stochastic network. To obtain the SN-MCP, we next develop the SN-UE and SN-SO models, respectively, and then explore the gap between them.

\section{Equilibrium Models under Stochastic Network}

3.1. Notations and Assumptions. Consider a road network $\mathbf{G}=(\mathbf{N}, \mathbf{A})$ with $\mathbf{N}$ being the set of nodes and $\mathbf{A}$ being the set of links, respectively. Let $\mathbf{W}$ be the set of all O-D pairs and $\mathbf{P}_{w}$ the set of all paths for O-D pair $w \in \mathbf{W}$. In the following, for consistency, random variables are expressed in upper-case letters and lower-case letters are used for the mean values:

$\mathrm{Q}_{w}:$ travel demand between O-D pair $w \in \mathbf{W}$;

$q_{w}$ : mean travel demand between O-D pair $w \in \mathbf{W}$;

$\varepsilon_{w}^{q}$ : variance of demand between O-D pair $w \in \mathbf{W}$;

$F_{k}^{w}:$ traffic flow on path $k \in \mathbf{P}_{w}$;

$f_{k}^{w}$ : mean traffic flow on path $k \in \mathbf{P}_{w}$;

$\mathbf{f}$ : vector of mean path flow, $\mathbf{f}=\left\{f_{k}^{w}\right\}$;

$\varepsilon_{k}^{w, f}$ : variance of traffic flow on path $k \in \mathbf{P}_{w}$;

$V_{a}:$ traffic flow on link $a \in \mathbf{A}$;

$v_{a}$ : mean traffic flow on link $a \in \mathbf{A}$; 
$\mathbf{v}$ : vector of mean link flow, $\mathbf{v}=\left\{v_{a}\right\}$;

$\varepsilon_{a}^{v}$ : variance of traffic flow on link $a \in \mathbf{A}$;

$T_{a}$ : travel time on link $a \in \mathbf{A}$;

$t_{a}:$ mean travel time on link $a \in \mathbf{A}$;

$\mathbf{t}$ : vector of mean link travel time, $\mathbf{t}=\left\{t_{a}\right\}$;

$C_{k}^{w}$ : travel time on path $k \in \mathbf{P}_{w}$;

$c_{k}^{w}$ : mean travel time on path $k \in \mathbf{P}_{w}$;

c: vector of mean path travel time, $\mathbf{c}=\left\{c_{k}^{w}\right\}$;

$\pi_{w}$ : minimum mean travel time between O-D pair $w \in$ W;

$\delta_{k, a}^{w}$ : indicator variable, 1 if path $k \in \mathbf{P}_{w}$ contains link $a \in$ A, 0 otherwise;

TT: total travel time, $\mathrm{TT}=\sum_{a \in \mathbf{A}} V_{a} T_{a}$.

The following basic assumptions are made throughout the paper, which are common in the literature of traffic equilibrium assignment with stochastic demand.

(A1) The O-D travel demands are assumed to be independently distributed. $\mathrm{VMR}_{w}$ is referred to as the variance to mean ratio of the stochastic demand in which $\mathrm{VMR}_{w}=\varepsilon_{w}^{q} / q_{w}$.

(A2) The path flow is assumed to be the product of path choice proportion and the O-D travel demand; that is, $F_{k}^{w}=p_{k}^{w} Q_{w}$, where $p_{k}^{w}=f_{k}^{w} / q_{w}$ that can be obtained from the model's output [21]. Then, it follows from (A1) that $F_{k}^{w}$ is also an independent random variable (r.v.) and follows the same statistical distribution as the O-D demand.

(A3) The VMRs of path flows are assumed to be equal to that of the corresponding O-D demand [7].

3.2. SN-UE Model. Due to the uncertainty of travel time travelers may consider the trade-off between the mean and variance of travel time when making their route choice decision [22]. Different travelers with different risk-taking attitude will consider different weight between the mean and variance. If the weight on variance is positive (negative), it indicates that travelers are "risk-averse" (risk-prone). If travelers are concerned with the mean travel time only, that is, the weight on variance is zero, they are regarded as "risk-neutral." In this study, we consider the case with the risk-neutral traveler only. The case with the risk prone/averse traveler can be extended in future.

The SN-UE proposed here is analogous to the Wardropian principle; that is, the equilibrium is reached when no riskneutral traveler can change his/her route unilaterally to reduce his/her average travel time which he/she experiences from day to day. This SN-UE condition can be mathematically stated as follows:

$$
\begin{array}{ll}
c_{k}^{w}=\pi_{w}, & \text { if } f_{k}^{w}>0 \\
c_{k}^{w} \geq \pi_{w}, & \text { if } f_{k}^{w}=0 .
\end{array} \quad \forall k \in \mathbf{P}_{w}, w \in \mathbf{W} .
$$

Such complementary conditions can be further reformulated as a VI problem: for any $\mathbf{f} \in \Omega_{\mathbf{f}}$, find $\mathbf{f}^{*} \in \Omega_{\mathbf{f}}$ such that

$$
\left(\mathbf{f}-\mathbf{f}^{*}\right)^{\mathrm{T}} \mathbf{c}^{*} \geq 0
$$

where $\Omega_{\mathbf{f}}$ is the feasible set of mean path flows defined as follows:

$$
\Omega_{\mathbf{f}}=\left\{\mathbf{f} \mid \mathbf{f} \geq \mathbf{0} ; \sum_{k \in \mathbf{P}_{w}} f_{k}^{w}=q_{w}, \forall w \in \mathbf{W}\right\} .
$$

Note that the link flow is the sum of the flows on all paths using the link, which gives

$$
\begin{aligned}
v_{a} & =E\left[V_{a}\right]=E\left[\sum_{w \in \mathbf{W}} \sum_{k \in \mathbf{P}_{w}} \delta_{k, a}^{w} F_{k}^{w}\right] \\
& =\sum_{w \in \mathbf{W}} \sum_{k \in \mathbf{P}_{w}} \delta_{k, a}^{w} E\left[F_{k}^{w}\right]=\sum_{w \in \mathbf{W}} \sum_{k \in \mathbf{P}_{w}} \delta_{k, a}^{w} f_{k}^{w} .
\end{aligned}
$$

In addition, the path travel time is the sum of the travel times on all links comprising the path, which gives

$$
\begin{aligned}
c_{k}^{w} & =E\left[C_{k}^{w}\right]=E\left[\sum_{a \in A} \delta_{k, a}^{w} T_{a}\right] \\
& =\sum_{a \in A} \delta_{k, a}^{w} E\left[T_{a}\right]=\sum_{a \in A} \delta_{k, a}^{w} t_{a} .
\end{aligned}
$$

More compactly, (5) and (6) can be written as $\mathbf{v}=\Delta \mathbf{f}$ and $\mathbf{c}=$ $\Delta^{\mathrm{T}} \mathbf{t}$, respectively, where $\Delta=\left\{\delta_{k, a}^{w}\right\}$ is the link-path incidence matrix. Substituting them into (3), we have

$$
\left(\mathbf{v}-\mathbf{v}^{*}\right)^{\mathrm{T}} \mathbf{t}^{*} \geq 0
$$

where the feasible region changes to $\Omega_{\mathbf{v}}$ as well. Consider

$$
\Omega_{\mathbf{v}}=\left\{\mathbf{v} \mid \mathbf{v}=\Delta \mathbf{f}, \mathbf{f} \geq \mathbf{0} ; \sum_{k \in \mathbf{P}_{w}} f_{k}^{w}=q_{w}, \forall w \in \mathbf{W}\right\} .
$$

3.3. SN-SO Model. For consistency, we also consider a riskneutral system manager here first. Let the expected total travel time be the measure of system performance. To achieve the best state, the SN-SO targets to minimize the expected total travel time. Based on Beckmann's formulation [23], the following mathematical program (MP) for SN-SO is defined:

$$
\min _{\mathbf{v} \in \Omega_{\mathbf{v}}} E[\mathrm{TT}]=E\left[\sum_{a \in \mathbf{A}} V_{a} T_{a}\right] .
$$

The optimality conditions of a MP can also be written as a VI problem if the objective function is continuously differentiable and the feasible region is closed and convex [24]. These two conditions are obviously satisfied by MP (9). Therefore, it can be reformulated as a VI problem: for any $\mathbf{v} \in \Omega_{\mathbf{v}}$, find $\mathbf{v}^{*} \in \Omega_{\mathbf{v}}$ such that

$$
\left(\mathbf{v}-\mathbf{v}^{*}\right)^{\mathrm{T}} \nabla_{\mathbf{v}} \mathbf{E}\left[\mathrm{TT}^{*}\right] \geq 0
$$

where $\nabla_{\mathbf{v}} \mathbf{E}\left[\mathrm{TT}^{*}\right]=\left\{\partial E\left[\mathrm{TT}^{*}\right] / \partial v_{a}^{*}\right\}$. 


\section{The SN-MCP and Its Calculation}

4.1. Derivation of the SN-MCP. Comparing (7) and (10), if travelers can realize that their travel cost is $\partial E[\mathrm{TT}] / \partial v_{a}$ but not $t_{a}$, the traffic flow pattern of SN-UE and SN-SO will be the same. However, the marginal external cost, that is, the gap between $\partial E[\mathrm{TT}] / \partial v_{a}$ and $t_{a}$, is often ignored by travelers. Thus, as in the deterministic network, to force travelers to notice such a cost, a toll that equates the gap on each link is charged.

Now we specifically focus on the link travel time function in a polynomial form:

$$
T_{a}=t_{a}\left(V_{a}\right)=\sum_{j=0}^{m} b_{j a} V_{a}^{j}, \quad \forall a \in \mathbf{A} .
$$

The power-law form of the commonly used BPR functions is a special case of (11). For other functional forms, a polynomial Taylor series approximation may be used to obtain the link travel time function in the form of (11).

Based on (11), the mean travel time on link $a \in \mathbf{A}$ is

$$
E\left[T_{a}\right]=E\left[\sum_{j=0}^{m} b_{j a} V_{a}^{j}\right]=\sum_{j=0}^{m} b_{j a} E\left[V_{a}^{j}\right],
$$

and the expected total travel time is

$$
E[\mathrm{TT}]=E\left[\sum_{a \in \mathbf{A}} \sum_{j=0}^{m} b_{j a} V_{a}^{j+1}\right]=\sum_{a \in \mathbf{A}} \sum_{j=0}^{m} b_{j a} E\left[V_{a}^{j+1}\right] .
$$

Therefore, the SN-MCP can be given as follows:

$$
\begin{aligned}
\mathrm{SN}-\mathrm{MCP} & =\frac{\partial E[\mathrm{TT}]}{\partial v_{a}}-E\left[T_{a}\right] \\
& =\sum_{j=0}^{m} b_{j a}\left(\frac{\partial E\left[V_{a}^{j+1}\right]}{\partial v_{a}}-E\left[V_{a}^{j}\right]\right) .
\end{aligned}
$$

Note that

$$
E\left[V_{a}^{j}\right]=\frac{\partial\left(E\left[V_{a}^{j}\right] E\left[V_{a}\right]\right)}{\partial v_{a}}-E\left[V_{a}\right] \frac{\partial E\left[V_{a}^{j}\right]}{\partial v_{a}} .
$$

Then replacing the term $E\left[V_{a}^{j}\right]$ in (14) with (15), we have

SN-MCP

$$
\begin{aligned}
& =\sum_{j=0}^{m} b_{j a}\left(\frac{\partial E\left[V_{a}^{j+1}\right]}{\partial v_{a}}-\frac{\partial\left(E\left[V_{a}^{j}\right] E\left[V_{a}\right]\right)}{\partial v_{a}}\right. \\
& \left.+E\left[V_{a}\right] \frac{\partial E\left[V_{a}^{j}\right]}{\partial v_{a}}\right) \\
& =v_{a} \frac{\partial E\left[T_{a}\right]}{\partial v_{a}}+\sum_{j=0}^{m} b_{j a}\left(\frac{\partial E\left[V_{a}^{j+1}\right]}{\partial v_{a}}-\frac{\partial\left(E\left[V_{a}^{j}\right] E\left[V_{a}\right]\right)}{\partial v_{a}}\right) \\
& =v_{a} \frac{\partial E\left[T_{a}\right]}{\partial v_{a}}+\sum_{j=0}^{m} b_{j a} \frac{\partial \operatorname{Cov}\left[V_{a}^{j}, V_{a}\right]}{\partial v_{a}},
\end{aligned}
$$

where the first term of the right-hand-side (RHS) of (16) is referred to as the average MCP and the second term is what the average MCP ignores. Obviously, as long as the covariance of $V_{a}^{j}$ and $V_{a}$ is a strictly monotone function of $v_{a}$, that is, $\partial \operatorname{Cov}\left[V_{a}^{j}, V_{a}\right] / \partial v_{a}>0$, the average MCP underestimates the real SN-MCP.

4.2. Calculation of the SN-MCP for a Special Case. To specifically quantify the SN-MCP, the exact value of each derivative in (16) should be calculated. In this subsection, we show how to derive the closed-form formulation of (16) for a special case with log-normal demands and an assumption of constant VMR across all O-D pairs. The constant VMR assumption is a little too strict in reality, but it will facilitate our following calculation and analysis. In the future study, we will try to relax this assumption.

The log-normal distribution is a positive and asymmetric distribution. The assumption that O-D demands follow lognormal distribution was adopted in many studies (see, e.g., $[7,25])$. Moreover, Uno et al. [26] used the empirical travel time data to validate the fact that the path travel times in their specific case study follow the log-normal distribution. To obtain the log-normal stochastic travel time in the proposed $\mathrm{SN}$, the assumption of the log-normal O-D demands is required.

Note that according to (A2), the path flows follow the log-normal distribution as well. Following Fenton [27], the summation of the log-normal r.v.s can be still estimated by a log-normal distribution. It shows that the link flow, which is the sum of related path flows, also follows the log-normal distribution, that is, $V_{a} \sim \operatorname{LN}\left(\mu_{a}^{v}, \sigma_{a}^{v}\right)$, where the distribution parameters $\mu_{a}^{v}$ and $\sigma_{a}^{v}$ are as follows:

$$
\begin{gathered}
\mu_{a}^{v}=\ln \left(v_{a}\right)-\frac{1}{2} \ln \left(1+\frac{\varepsilon_{a}^{v}}{\left(v_{a}\right)^{2}}\right), \\
\left(\sigma_{a}^{v}\right)^{2}=\ln \left(1+\frac{\varepsilon_{a}^{v}}{\left(v_{a}\right)^{2}}\right) .
\end{gathered}
$$

Any $j$ th moment of the log-normal link flow $V_{a}$ exists and can be calculated via the moment generating function. The general expression is given by the following:

$$
E\left[V_{a}^{j}\right]=\exp \left(j \mu_{a}^{v}+\frac{j^{2}\left(\sigma_{a}^{v}\right)^{2}}{2}\right)
$$

In addition to the assumption on statistical distribution of demands, the other assumption that the VMRs of demands are the same for all O-D pairs is required to allow for the closed-form calculation of the proposed SN-MCP, in which $E\left[V_{a}^{j}\right]$ and $\operatorname{Cov}\left[V_{a}^{j}, V_{a}\right]$ can be derived as a function of the mean link flow $v_{a}$.

Again utilizing the relationship between the link and path flows, we have

$$
\varepsilon_{a}^{v}=\operatorname{Var}\left[V_{a}\right]=\operatorname{Var}\left[\sum_{w \in \mathbf{W}} \sum_{k \in \mathbf{P}_{w}} \delta_{k, a}^{w} F_{k}^{w}\right]
$$




$$
=\sum_{w \in \mathbf{W}} \sum_{k \in \mathbf{P}_{w}}\left(\delta_{k, a}^{w}\right)^{2} \operatorname{Var}\left[F_{k}^{w}\right]=\sum_{w \in \mathbf{W}} \sum_{k \in \mathbf{P}_{w}} \delta_{k, a}^{w} \varepsilon_{k}^{w, f} .
$$

With (A3) and the constant VMR assumption, $\varepsilon_{k}^{w, f}=\mathrm{VMR}$. $f_{k}^{w}$. Therefore, it follows from (19) and (5) that the variance of link flow can be further reduced to

$$
\varepsilon_{a}^{v}=\mathrm{VMR} \cdot \sum_{w \in \mathbf{W}} \sum_{k \in \mathbf{P}_{w}} \delta_{k, a}^{w} f_{k}^{w}=\mathrm{VMR} \cdot v_{a} .
$$

Now let us combine (17), (18), and (20) together and perform some manipulations; then it yields that

$$
\begin{aligned}
E\left[V_{a}^{j}\right]= & v_{a}^{j}\left(\sqrt{1+\frac{\mathrm{VMR}}{v_{a}}}\right)^{j^{2}-j}, \\
\operatorname{Cov}\left[V_{a}^{j}, V_{a}\right]= & E\left[V_{a}^{j+1}\right]-E\left[V_{a}^{j}\right] E\left[V_{a}\right] \\
= & v_{a}^{j+1}\left(\sqrt{1+\frac{\mathrm{VMR}}{v_{a}}}\right)^{j^{2}+j} \\
& -v_{a}^{j+1}\left(\sqrt{1+\frac{\mathrm{VMR}}{v_{a}}}\right)^{j^{2}-j} .
\end{aligned}
$$

Using notation $s_{a}=\sqrt{1+\mathrm{VMR} / v_{a}}$ to simplify the formulation, we have

$$
\begin{aligned}
& \frac{\partial \operatorname{Cov}\left[V_{a}^{j}, V_{a}\right]}{\partial v_{a}} \\
& =(j+1) v_{a}^{j}\left[s_{a}^{j^{2}+j}-s_{a}^{j^{2}-j}\right] \\
& \quad+\left(\frac{1-s_{a}^{2}}{2 s_{a}^{2}}\right) v_{a}^{j}\left[\left(j^{2}+j\right) s_{a}^{j^{2}+j}-\left(j^{2}-j\right) s_{a}^{j^{2}-j}\right] .
\end{aligned}
$$

Furthermore,

$$
\begin{aligned}
\frac{\partial E\left[T_{a}\right]}{\partial v_{a}} & =\sum_{j=0}^{m} b_{j a} \frac{\partial E\left[V_{a}^{j}\right]}{\partial v_{a}} \\
& =\sum_{j=0}^{m} b_{j a} v_{a}^{j-1} s_{a}^{j^{2}-j}\left[j+\left(j^{2}-j\right) \cdot \frac{1-s_{a}^{2}}{2 s_{a}^{2}}\right] .
\end{aligned}
$$

By substituting (22) and (23) into (16), the value of the SNMCP can be determined as long as the mean link flows under the SN-SO are known. The closed-form formulation can also be derived from other statistical distributions of travel demand. In the numerical test part, the case with normal distribution will be checked as well.

\section{Trial-and-Error Implementation of SN-MCP}

As mentioned, the trial-and-error methods developed before are all based on the assumption of deterministic traffic state.
The precise deterministic UE link flows are assumed to be observable and are used to adjust the toll levels in each trial. However, just considering the observation errors, such an assumption cannot be truly realized in practice, not to mention the variability of link flows and travel times due to the stochastic demand. If one observes the link flow on a section of highway between a certain time of day and over a time period (e.g., one month), it is obvious that the collected flow count data are random. Due to the uncertainty of traffic flow information, we cannot arbitrarily pick up one day's observation data to refer to in the toll updating scheme. Instead the mean of the data collected over a time period is preferred to be utilized. However, it should be noted that when the mean values are used in the toll updating scheme, the MCP determination formula as shown in (16) (i.e., SN$\mathrm{MCP}$ ) is different with the original MCP replaced by the mean value. Therefore, in this section, we are intent on revising the trial-and-error procedure presented by Yang et al. [5] to allow it to proceed in the studied SN case as well.

Here assume that the stochastic travel demand follows some statistical distribution, but the distribution parameters are unknown and not easy to be estimated. In other words, the mean and variance of each $\mathrm{O}-\mathrm{D}$ demand are not available. Under this situation, it is impossible to find the equilibrium flow pattern and thereby obtain the marginal-cost tolls by directly solving the related mathematical problem. The obstacle that we meet now is the same as that in the case of deterministic network. To remove the influence of the limited information, we turn to the trial-and-error idea again to propose a method that iteratively calculates the marginalcost tolls based on the observable stochastic link flows. The detailed implementation procedure is presented below.

Step 0 (initialization). Let $\left\{v_{a}^{(0)}, a \in \mathbf{A}\right\}$ be an initial set of feasible mean link flows. Set $k=0$.

Step 1 (estimate link tolls). For each link $a \in \mathbf{A}$, calculate the current link toll $\tau_{a}^{(k)}$ by

$$
\tau_{a}^{(k)}=v_{a}^{(k)} \frac{\partial E\left[T_{a}^{(k)}\right]}{\partial v_{a}^{(k)}}+\sum_{j=0}^{m} b_{j a} \frac{\partial \operatorname{Cov}\left[\left(V_{a}^{(k)}\right)^{j}, V_{a}^{(k)}\right]}{\partial v_{a}^{(k)}} .
$$

Step 2 (observe link flows). After imposition of the link tolls given by (24) on a network, observe and collect the link count data over a period of time. Then, compute the mean and variance of the collected link flows via data fitting. Let $\left\{\bar{v}_{a}^{(k)}, a \in \mathbf{A}\right\}$ denote the estimated mean value.

Step 3 (check convergence). If $\left\|\overline{\mathbf{v}}^{(k)}-\mathbf{v}^{(k)}\right\| /\left\|\mathbf{v}^{(k)}\right\|<\varepsilon$, then stop. Otherwise, go to Step 4.

Step 4 (update link flows). Set

$$
v_{a}^{(k+1)}=v_{a}^{(k)}+\alpha^{(k)}\left(\bar{v}_{a}^{(k)}-v_{a}^{(k)}\right), \quad a \in \mathbf{A},
$$

and $k:=k+1$; go to Step 1 .

In the procedure above, " $\|\cdot\|$ " denotes the Euclidean norm; $\varepsilon$ is a positive number of convergence tolerance; $\left\{\alpha^{(k)}\right\}$ is a 
sequence of predetermined step sizes and it must satisfy the following three conditions:

$$
0<\alpha^{(k)} \leq 1, \quad \sum_{k=1}^{\infty} \alpha^{(k)}=+\infty, \quad \sum_{k=1}^{\infty}\left(\alpha^{(k)}\right)^{2}<+\infty .
$$

A typical sequence of $\alpha^{(k)}$ is $\alpha^{(k)}=1 / k$.

Remark 1. In Section 4, the SN-MCP given by (24) was specifically quantified for a special case with log-normal demands and an assumption of constant VMR across all O$\mathrm{D}$ pairs. From the previous analysis, it is known that, for that special case, each trial toll pattern in Step 1 could be calculated when the mean link flows and the VMR of O-D demands are available. Since the mean and variance of all stochastic O-D demands are assumed to be unknown in practice, the VMR of O-D demands is unknown as well. However, under the assumptions made before, the VMR of O-D demands could be obtained by calculating the VMR of link flows according to $(20)$. When implementing the above algorithm in reality, both the mean and the variance of each UE link flow can be estimated by the collected link traffic count data. The only question is that the variance to mean ratio (VMR) of each link flow is probably not the same exactly due to random errors. Therefore, in the following numerical experiments, we take the average of all VMRs of link flows as the required constant VMR in the algorithm.

Remark 2. At each iteration of the above algorithm, we will fit a series of observed link flow data into a suitable probability distribution. Then, the parameters of the distribution, that is, the mean and variance of UE link flows, can be obtained. The "dfittool" in MATLAB will be utilized to fit distributions in the following numerical experiments.

5.1. Convergence of the Trial-and-Error Implementation. In order to prove the convergence of the above-presented trialand-error method, we provide the following two important propositions, which are similar to that in Yang et al. [5].

Proposition 3. If $\left\|\overline{\mathbf{v}}^{(k)}-\mathbf{v}^{(k)}\right\|=0$ at the convergent point, then $\left\{v_{a}^{(k)}, a \in \mathbf{A}\right\}$ is the mean SN-UE link flow pattern and $\left\{\tau_{a}^{(k)}, a \in \mathbf{A}\right\}$ is the corresponding optimal link toll pattern.

Proof. According to the above algorithm, after the toll pattern $\boldsymbol{\tau}^{(k)}=\left\{\tau_{a}^{(k)}, a \in \mathbf{A}\right\}$ as shown in (24) is implemented, the revealed mean link flow pattern, $\overline{\mathbf{v}}^{(k)}=\left\{\bar{v}_{a}^{(k)}, a \in \mathbf{A}\right\}$, is the solution of the following VI problem:

$$
\left(\mathbf{v}-\overline{\mathbf{v}}^{(k)}\right)^{\mathrm{T}}\left(\overline{\mathbf{t}}^{(k)}+\boldsymbol{\tau}^{(k)}\right) \geq 0, \quad \forall \mathbf{v} \in \Omega
$$

If $\left\|\overline{\mathbf{v}}^{(k)}-\mathbf{v}^{(k)}\right\|=0$, then $\bar{v}_{a}^{(k)}=v_{a}^{(k)}$ for all link $a$. Hence

$$
\overline{\mathbf{t}}^{(k)}+\boldsymbol{\tau}^{(k)}=\mathbf{t}^{(k)}+\boldsymbol{\tau}^{(k)}=\nabla_{\mathbf{v}} \mathbf{E}\left[\mathrm{TT}^{(k)}\right]
$$

which means $\overline{\mathbf{v}}^{(k)}$, or say $\mathbf{v}^{(k)}$, is already the optimal solution of VI problem (10). That is, the toll pattern $\boldsymbol{\tau}^{(k)}$ now is the optimal one in the sense of first-best pricing.
Proposition 4. If the mean link travel cost $\mathbf{t}$ is strongly monotone with respect to the mean link flow $\mathbf{v}$, that is, there exists a positive constant $\rho$ such that, for any distinct $\mathbf{v}_{1} \in \Omega_{\mathbf{v}}$ and $\mathbf{v}_{2} \in \Omega_{\mathbf{v}}$, we have

$$
\left(\mathbf{t}_{1}-\mathbf{t}_{2}\right)^{\mathrm{T}}\left(\mathbf{v}_{1}-\mathbf{v}_{2}\right) \geq \rho\left\|\mathbf{v}_{1}-\mathbf{v}_{2}\right\|^{2}
$$

then the vector $\left(\overline{\mathbf{v}}^{(k)}-\mathbf{v}^{(k)}\right)$ is a feasible descent direction of the objective function of MP (9) at $\mathbf{v}^{(k)}$.

Proof. Note that the gradient of objective function (9) is given as follows:

$$
\nabla_{\mathbf{v}} \mathrm{E}\left[\mathrm{TT}^{(k)}\right]=\mathbf{t}^{(k)}+\boldsymbol{\tau}^{(k)}
$$

Therefore,

$$
\begin{aligned}
\left(\overline{\mathbf{v}}^{(k)}-\mathbf{v}^{(k)}\right)^{\mathrm{T}} \nabla_{\mathbf{v}} \mathbf{E}\left[\mathrm{TT}^{(k)}\right]= & \left(\overline{\mathbf{v}}^{(k)}-\mathbf{v}^{(k)}\right)^{\mathrm{T}}\left(\mathbf{t}^{(k)}+\boldsymbol{\tau}^{(k)}\right) \\
= & \left(\overline{\mathbf{v}}^{(k)}-\mathbf{v}^{(k)}\right)^{\mathrm{T}}\left(\mathbf{t}^{(k)}-\overline{\mathbf{t}}^{(k)}\right) \\
& +\left(\overline{\mathbf{v}}^{(k)}-\mathbf{v}^{(k)}\right)^{\mathrm{T}}\left(\overline{\mathbf{t}}^{(k)}+\boldsymbol{\tau}^{(k)}\right) .
\end{aligned}
$$

From (29), the first term of (31) is less than or equal to $-\rho\left\|\overline{\mathbf{v}}^{(k)}-\mathbf{v}^{(k)}\right\|^{2}$. From (27), the second term of (31) is nonpositive. Thus,

$$
\left(\overline{\mathbf{v}}^{(k)}-\mathbf{v}^{(k)}\right)^{\mathrm{T}} \nabla_{\mathbf{v}} \mathbf{E}\left[\mathrm{TT}^{(k)}\right] \leq-\rho\left\|\overline{\mathbf{v}}^{(k)}-\mathbf{v}^{(k)}\right\|^{2} .
$$

It is obvious that, as long as $\overline{\mathbf{v}}^{(k)} \neq \mathbf{v}^{(k)}$, we have $\left(\overline{\mathbf{v}}^{(k)}-\right.$ $\left.\mathbf{v}^{(k)}\right)^{\mathrm{T}} \nabla_{\mathbf{v}} \mathbf{E}\left[\mathrm{TT}^{(k)}\right]<0$, which indicates that the vector $\left(\overline{\mathbf{v}}^{(k)}-\right.$ $\left.\mathbf{v}^{(k)}\right)$ is a descent direction.

Now let us illustrate that the proposed iterative procedure does converge; that is, $\mathbf{v}^{(k)} \rightarrow \mathbf{v}^{*}$ and $\boldsymbol{\tau}^{(k)} \rightarrow \boldsymbol{\tau}^{*}$ when $k \rightarrow$ $\infty$. Since the separable link travel time function $t_{a}\left(V_{a}\right)$ is assumed, the Hessian matrix of objective function (9) must be a diagonal matrix. Let $E_{a}^{\prime \prime}[\mathrm{TT}]$ represent its diagonal element; then

$$
\begin{gathered}
\left(\overline{\mathbf{v}}^{(k)}-\mathbf{v}^{(k)}\right)^{\mathrm{T}} \nabla_{\mathbf{v}}^{2} \mathbf{E}[\mathrm{TT}]\left(\overline{\mathbf{v}}^{(k)}-\mathbf{v}^{(k)}\right) \\
=\sum_{a \in \mathbf{A}} E_{a}^{\prime \prime}[\mathrm{TT}]\left(\bar{v}_{a}^{(k)}-v_{a}^{(k)}\right)^{2} .
\end{gathered}
$$

The RHS of (33) is always bounded for the bounded link travel time functions. Combining this conclusion with Proposition 4 and the conditions for choosing the step size sequence, all convergence requirements regarding the method of successive average (MSA) are satisfied [28]. Thus, the convergence of the proposed trial-and-error algorithm is followed.

5.2. Analysis of the Sampling Error on the Trial-and-Error Implementation. As mentioned above, we present a theoretical proof on the convergence of the proposed trial-anderror procedure. All the propositions are shown to hold with the true mean link flows under SN-UE. In real application, 
however, the true mean link flows also cannot be exactly obtained. The reason is that when the trial-and-error procedure is executed in practice, the link flow data can only be observed and collected over a limited period of time (e.g., one month). Hence, the mean link flows calculated from the limited sampling data are generally not equal to their true values. In other words, at each iteration, only an approximate mean link flow pattern can be used to update the trial tolls. Then a question emerges: will such an approximation affect the convergence of the trial-and-error procedure or the correctness of the convergent result?

Fortunately, the iterative algorithm we explored here is in essential the method of successive average. The convergence requirements of such a method can guarantee the sequence of solutions to converge to the minimum even if the search direction is a descent vector only on the average. For example, when the MSA is applied to solve the SUE problem, it sometimes requires a Probit stochastic network loading via using Monte-Carlo simulation to determine the descent direction. If such a technique is resorted, then no matter how many times the simulation procedure is repeated, the resulting averaged link flows are not the actual ones. The direction vectors that are produced by such simulations are thereby random direction. However, even in this case, the MSA algorithm still can converge as long as an unbiased estimate of the direction is utilized.

Now we encounter the same situation when simulating the mean values of the link flows. As mentioned before, regardless of how long the observation period is allowed and how many the sampling data are collected, the true mean value cannot be accurately computed but estimated. Therefore, each time after the trial toll charge is imposed on the network, the estimated mean SN-UE link flow pattern $\overline{\mathbf{v}}^{(k)}$ is in fact a random variable. That is to say, the descent direction vector $\left(\overline{\mathbf{v}}^{(k)}-\mathbf{v}^{(k)}\right)$ is random as well.

Using the simplest step size sequence that satisfies conditions (26), $\left\{\alpha^{(k)}=1 / k\right\}$, then

$$
\mathbf{v}^{(k+1)}=\mathbf{v}^{(k)}+\frac{1}{k}\left(\overline{\mathbf{v}}^{(k)}-\mathbf{v}^{(k)}\right) .
$$

This relation can be rewritten as follows: as its name, the method of successive average, means:

$$
\mathbf{v}^{(k+1)}=\frac{1}{k} \sum_{l=1}^{k} \overline{\mathbf{v}}^{(l)} .
$$

Thus the variance of each component of $\mathbf{v}^{(k+1)}$ is given by

$$
\operatorname{Var}\left(v_{a}^{(k+1)}\right)=\frac{1}{k^{2}} \sum_{l=1}^{k} \operatorname{Var}\left(\bar{v}_{a}^{(l)}\right) .
$$

At each iteration, since the revealed link flow $\bar{v}_{a}^{(l)}$ is always below the sum of all O-D flows, the variance of $\bar{v}_{a}^{(l)}$ is bounded by some value; that is, $\operatorname{Var}\left(\bar{v}_{a}^{(l)}\right)<\sigma^{2}<\infty, \forall l$. Therefore,

$$
\operatorname{Var}\left(v_{a}^{(k+1)}\right)<\frac{1}{k^{2}} \sum_{l=1}^{k} \sigma^{2} .
$$

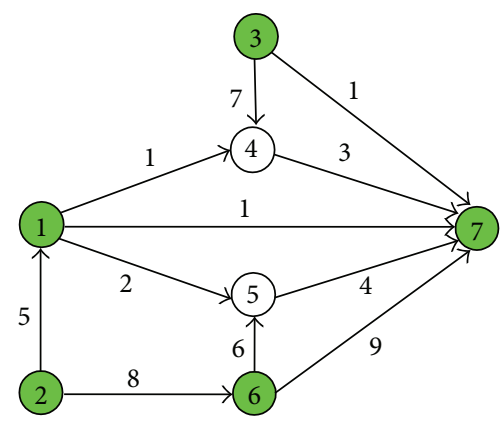

Figure 1: Road network used for Example 1.

TABLE 1: Link cost parameters for Example 1.

\begin{tabular}{lccc}
\hline Link & $t_{a}^{0}$ & $b_{a}$ & $c_{a}$ \\
\hline 1 & 6 & 0.90 & 200 \\
2 & 5 & 0.75 & 200 \\
3 & 6 & 0.90 & 200 \\
4 & 7 & 1.05 & 200 \\
5 & 6 & 0.90 & 100 \\
6 & 1 & 0.15 & 100 \\
7 & 5 & 0.75 & 150 \\
8 & 10 & 1.50 & 150 \\
9 & 11 & 1.65 & 200 \\
10 & 11 & 1.65 & 200 \\
11 & 15 & 2.25 & 200 \\
\hline
\end{tabular}

Obviously, the RHS of (37) approaches zero as $k$ grows, meaning that the variance of $v_{a}^{(k+1)}$ approaches zero as the algorithm progresses. This further indicates that the variance of trial toll pattern $\boldsymbol{\tau}^{(k+1)}$ updated by using $\mathbf{v}^{(k+1)}$ approaches zero too. In terms of these properties, it can be ensured that the convergence of the proposed iterative procedure will not be affected no matter how accurate the simulation is at each iteration.

\section{Numerical Tests}

Example 1. The test network for Example 1 is taken from Yang et al. [5] as shown in Figure 1, which has 7 nodes, 11 links, and 4 O-D pairs.

In this example, the BPR link cost function, that is,

$$
t_{a}\left(V_{a}\right)=t_{a}^{0}+b_{a}\left(\frac{V_{a}}{c_{a}}\right)^{n_{a}}
$$

is adopted with $n_{a}=4$ for all link $a$. The other link cost parameters (e.g., the free-flow travel time $t_{a}^{0}$ and the capacity $c_{a}$ of each link) are given in Table 1.

For each O-D pair, the mean of the log-normal demand is shown in Table 2. It is worth noting that the reason we give the mean values here is that, in numerical experiments, the SN-UE flow pattern cannot be observed. Thus, the mean values are purely used for generating the observed link flows, 
TABLE 2: Mean of the log-normal demand for Example 1.

\begin{tabular}{lc}
\hline O-D pair & $q_{w}$ \\
\hline $1 \rightarrow 7$ & 420 \\
$2 \rightarrow 7$ & 350 \\
$3 \rightarrow 7$ & 350 \\
$6 \rightarrow 7$ & 280 \\
\hline
\end{tabular}

which is not necessary for updating link tolls in the real implementation of trial-and-error method.

In this example, we examine three VMR levels; that is, $\mathrm{VMR}=0,20$, and 40 . When $\mathrm{VMR}=0$, it means no uncertainty on the network, that is, deterministic network. Here the values of VMR are given in order to generate the true mean link flows and marginal cost tolls, which will be used to compare with the estimated results. The true mean link flows and tolls calculated by the MSA are summarized in Table 3. It can be seen that the true SN-MCP tolls are higher than those from the average and original MCP calculations. Table 4 shows the expected total travel time at different VMR levels. The "Improv." column shows the percentage of improvement of the expected total travel time (from the SN$\mathrm{UE})$ as compared to the SN-SO case; that is,

$$
\text { Improv. (case) }=\frac{E\left(\mathrm{TT}_{\mathrm{SN}-\mathrm{UE}}\right)-E\left(\mathrm{TT}_{\text {case }}\right)}{E\left(\mathrm{TT}_{\mathrm{SN}-\mathrm{UE}}\right)-E\left(\mathrm{TT}_{\mathrm{SN}-\mathrm{SO}}\right)} \times 100 \% \text {. }
$$

Figure 2 plots the percentage improvements shown in Table 4. From the figure, it is clear that the improvement of the expected total travel time made by the average MCP and the original MCP schemes are lower than those of the SN-MCP scheme. In particular, when the network is highly uncertain (e.g., VMR = 40 in this example), the origin MCP schemes instead increases the expected total travel time compared to the no toll case.

The estimated mean link flows and SN-SO tolls obtained through the trial-and-error algorithm are summarized in Table 5 . The iterative procedure initially starts from a uniform toll scheme; that is, $\tau_{a}=15$, for all link $a$. The step size $\alpha^{(k)}=$ $1 / k$ and convergence tolerance $\varepsilon=0.005$ are utilized in the trial-and-error algorithm. With different VMR levels, Figure 3 illustrates the nice convergence of the iterative procedure. All the trial-and-error processes converge in 10 iterations.

If we do not use the SN-MCP formula to calculate the trial tolls in Step 1 as shown in (24) but refer to the average MCP or original MCP, the trial-and-error procedure cannot converge to the true optimal tolls that achieves SN-SO. Figure 4 shows this phenomenon for $\mathrm{VMR}=20$.

Example 2. The test network for Example 2 is taken from Sumalee et al. $[11,12]$ as shown in Figure 5, which has 7 nodes, 18 links, and 6 O-D pairs. This example again takes the BPR link cost function with $n_{a}=4$ for all link $a$. Table 6 gives the other link cost parameters adopted in this example. The mean values of the stochastic demands are shown in Table 7.

Here the numerical test is based on another statistical distribution of O-D travel demand, normal distribution, which is also widely adopted in the literature (e.g., [21, 29, 30]). From this example, it is found that some of mean link flows could be zero when arriving at the equilibrium, for example, the SN-UE flow pattern with no toll. The log-normal distribution, however, excludes this instance. Under the assumptions that the travel demand follows a normal distribution and the path flow has the same kind of distribution as the demand, the link flow, which is the sum of path flows, also follows normal distribution; that is, $V_{a} \sim N\left(v_{a}, \varepsilon_{a}^{v}\right)$.

Using the method of moment generating function, the mean link travel time can be expressed explicitly in terms of the mean and variance of the stochastic link flow. And further noting the relationship between the mean and variance as shown in (20), we have

$$
\begin{aligned}
E\left[T_{a}\right] & =t_{a}^{0}+\frac{b_{a}}{c_{a}^{4}} E\left[V_{a}^{4}\right] \\
& =t_{a}^{0}+\frac{b_{a}}{c_{a}^{4}}\left[v_{a}^{4}+6 v_{a}^{2} \varepsilon_{a}^{v}+3\left(\varepsilon_{a}^{v}\right)^{2}\right] \\
& =t_{a}^{0}+\frac{b_{a}\left[v_{a}^{4}+6 \mathrm{VMR}_{a}^{3}+3 \mathrm{VMR}^{2} v_{a}^{2}\right]}{c_{a}^{4}}
\end{aligned}
$$

Similarly, we can compute $E[\mathrm{TT}]$, which is

$$
E[\mathrm{TT}]=t_{a}^{0} v_{a}+\frac{b_{a}\left[v_{a}^{5}+10 \mathrm{VMR} v_{a}^{4}+15 \mathrm{VMR}^{2} v_{a}^{3}\right]}{c_{a}^{4}}
$$

Therefore,

$$
\frac{\partial E[\mathrm{TT}]}{\partial v_{a}}=t_{a}^{0}+\frac{b_{a}\left[5 v_{a}^{4}+40 \mathrm{VMR} v_{a}^{3}+45 \mathrm{VMR}^{2} v_{a}^{2}\right]}{c_{a}^{4}} .
$$

Then,

$$
\begin{aligned}
\mathrm{SN}-\mathrm{MCP} & =\frac{\partial E[\mathrm{TT}]}{\partial v_{a}}-E\left[T_{a}\right] \\
& =\frac{b_{a}\left[4 v_{a}^{4}+34 \mathrm{VMR} v_{a}^{3}+42 \mathrm{VMR}^{2} v_{a}^{2}\right]}{c_{a}^{4}} .
\end{aligned}
$$

Again, taking the step size $\alpha^{(k)}=1 / k$ and setting the convergence tolerance $\varepsilon=0.01$ in the trial-and-error algorithm, we estimate the optimal toll charges under stochastic demands with $\mathrm{VMR}=100$. The estimated results and the true results are both presented in Table 8 for comparison. Figure 6 again exhibits the nice convergence of the iterative procedure.

\section{Conclusions}

This study discussed the first-best marginal cost pricing and its trial-and-error implementation under a stochastic network that explicitly considers the demand uncertainty. In the paper, we derived the SN-MCP by reinvestigating the real gap between the marginal social and private costs in terms of stochastic traffic flows. There are two terms involved in the SN-MCP when travelers are assumed to be risk-neutral. The first term can be regarded as the simple modification of the 


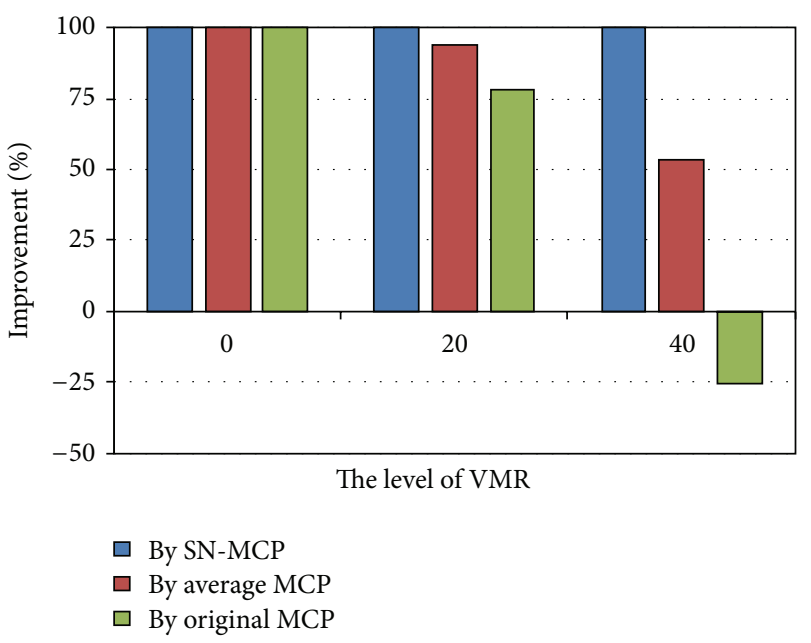

FIGURE 2: Improvement of system performance by different MCP schemes.

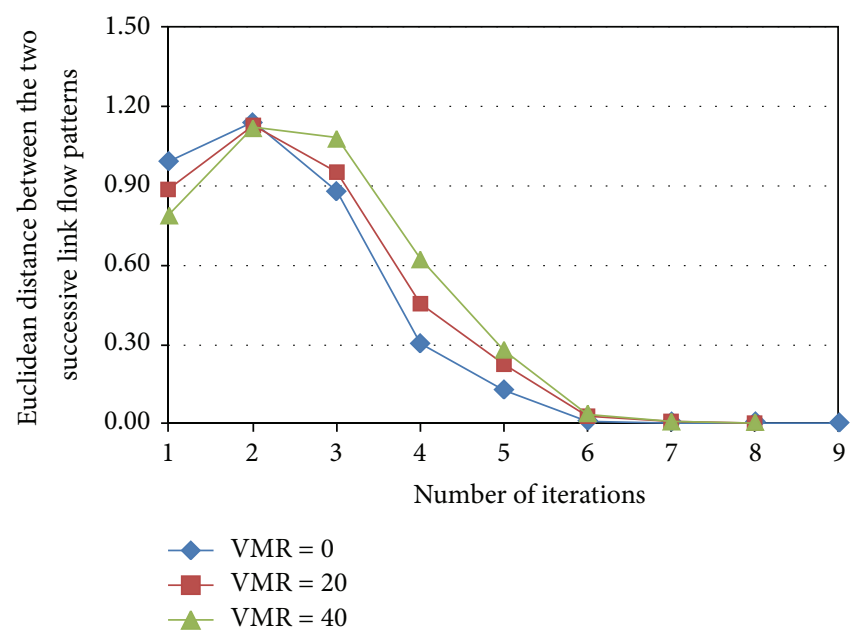

FIGURE 3: Convergence of the trial-and-error procedure for SN-MCP with different VMR levels.

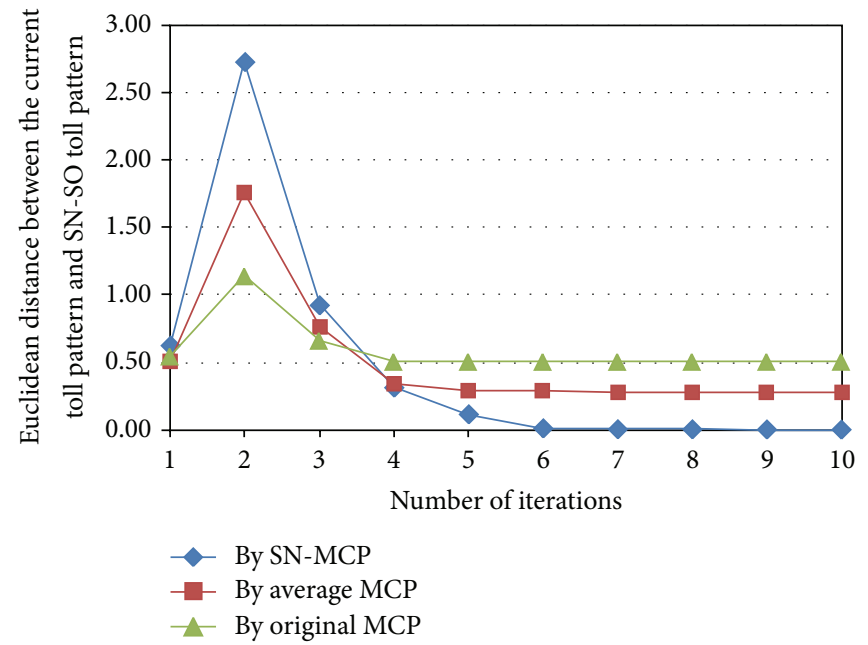

FIGURE 4: Convergence of the trial-and-error procedure by different MCP schemes. 
TABLE 3: True mean link flows and tolls under SN-SO.

\begin{tabular}{|c|c|c|c|c|c|c|}
\hline \multirow{3}{*}{ Link } & \multicolumn{2}{|c|}{$\mathrm{VMR}=0$} & \multicolumn{2}{|c|}{$\mathrm{VMR}=20$} & \multicolumn{2}{|c|}{$\mathrm{VMR}=40$} \\
\hline & Mean flow & Toll & Mean flow & Toll & Mean flow & Toll \\
\hline & $v_{a}$ & $\tau_{a}$ & $v_{a}$ & $\tau_{a}$ & $v_{a}$ & $\tau_{a}$ \\
\hline 1 & 212.2 & 4.6 & 207.9 & 9.0 & 204.8 & 16.9 \\
\hline 2 & 119.7 & 0.4 & 121.9 & 1.4 & 123.6 & 4.0 \\
\hline 3 & 301.7 & 18.6 & 300.7 & 31.6 & 299.3 & 50.9 \\
\hline 4 & 305.4 & 22.8 & 306.0 & 39.1 & 306.1 & 63.6 \\
\hline 5 & 158.5 & 22.7 & 153.4 & 54.9 & 147.7 & 117.0 \\
\hline 6 & 185.7 & 7.1 & 184.0 & 16.2 & 182.6 & 33.2 \\
\hline 7 & 89.5 & 0.4 & 92.8 & 2.1 & 94.5 & 7.2 \\
\hline 8 & 191.5 & 16.0 & 196.6 & 39.6 & 202.3 & 86.3 \\
\hline 9 & 285.8 & 27.5 & 292.6 & 52.6 & 299.7 & 93.7 \\
\hline 10 & 260.5 & 19.0 & 257.2 & 33.7 & 255.5 & 58.1 \\
\hline 11 & 246.6 & 20.8 & 243.5 & 38.2 & 239.4 & 65.6 \\
\hline
\end{tabular}

TABLE 4: Comparison of the expected total time under different VMR levels.

\begin{tabular}{|c|c|c|c|c|c|c|c|c|}
\hline \multirow{2}{*}{ VMR } & \multicolumn{2}{|c|}{ SN-UE (toll free) } & \multicolumn{2}{|c|}{ SN-SO (SN-MCP) } & \multicolumn{2}{|c|}{ Average MCP } & \multicolumn{2}{|c|}{ Original MCP } \\
\hline & $E(\mathrm{TT})$ & Improv. & $E(\mathrm{TT})$ & Improv. & $E(\mathrm{TT})$ & Improv. & $E(\mathrm{TT})$ & Improv. \\
\hline 0 & 29098 & $0.0 \%$ & 28919 & $100.0 \%$ & 28919 & $100.0 \%$ & 28919 & $100.0 \%$ \\
\hline 20 & 40994 & $0.0 \%$ & 40838 & $100.0 \%$ & 40848 & $93.8 \%$ & 40873 & $78.0 \%$ \\
\hline 40 & 65752 & $0.0 \%$ & 65593 & $100.0 \%$ & 65666 & $53.6 \%$ & 65793 & $-25.8 \%$ \\
\hline
\end{tabular}

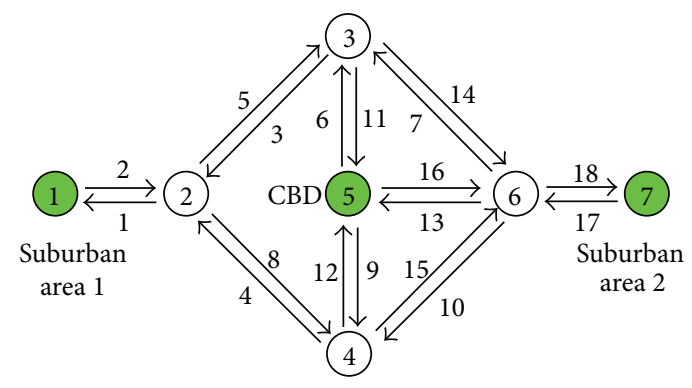

FIGURE 5: Road network used for Example 2.

original MCP; that is, the originally deterministic link flows and travel times are substituted by their mean values. The second term is the additional term related to the variability of travel demand, which is often ignored.

Given the specific statistical distribution of random demands and the assumption of constant VMR across all O-D pairs, the closed-form formulation of SN-MCP can be calculated with the information of true mean values of demands. In reality, when this information is not available, a revised trial-and-error method was proposed for practical implementation. The trial tolls are computed based on the formula of SN-MCP at each step but not the original MCP. Though the mean link flows cannot be precisely obtained due to the limited sample size, it has been shown that the sampling error will not affect the convergence of the proposed trial-and-error procedure. The convergence and effectiveness of this evolutionary implementation were not only proved

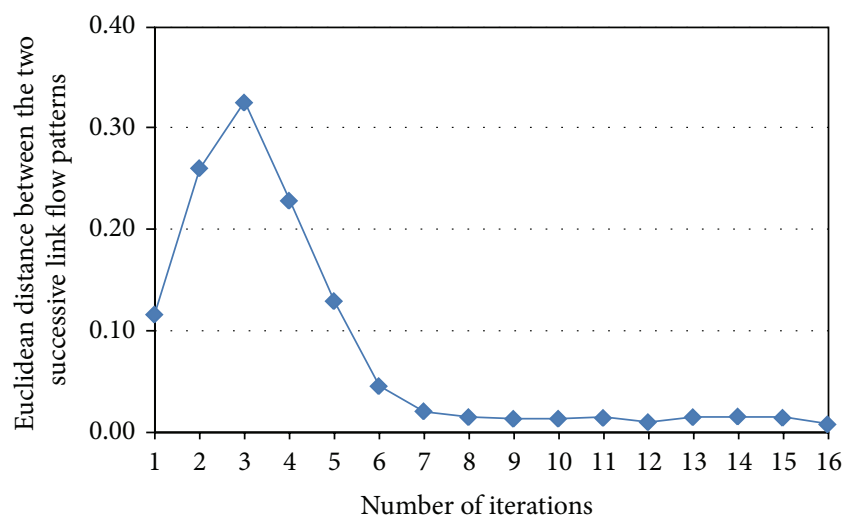

FIGURE 6: Convergence of the trial-and-error procedure for SNMCP.

theoretically but also examined by two numerical tests. The first one was carried out under the assumption of log-normal demand, while the second one assumed the normal demand.

This paper only investigated the traffic assignment and road pricing with the case of inelastic demand noting that the mean of stochastic demand is fixed. If the level of average demand is influenced by the travel cost of the trip, that is, with the case of elastic demand, the SN-MCP we derived in this study is still applicable to drive the link flow pattern towards a SN-SO, where the SN-SO aims to maximize the expected social welfare. At the same time, the convergence of the revised trial-and-error algorithm can be guaranteed as well. The whole analysis will be very similar as that in this study if 
TABLE 5: Estimated mean link flows and tolls under SN-SO.

\begin{tabular}{|c|c|c|c|c|c|c|}
\hline \multirow{3}{*}{ Link } & \multicolumn{2}{|c|}{$\mathrm{VMR}=0$} & \multicolumn{2}{|c|}{$\mathrm{VMR}=20$} & \multicolumn{2}{|c|}{$\mathrm{VMR}=40$} \\
\hline & Mean flow & Toll & Mean flow & Toll & Mean flow & Toll \\
\hline & $v_{a}$ & $\tau_{a}$ & $v_{a}$ & $\tau_{a}$ & $v_{a}$ & $\tau_{a}$ \\
\hline 1 & 213.4 & 4.5 & 207.9 & 9.0 & 203.8 & 16.9 \\
\hline 2 & 117.7 & 0.4 & 121.2 & 1.4 & 123.3 & 4.0 \\
\hline 3 & 301.9 & 18.7 & 299.7 & 31.5 & 299.1 & 51.1 \\
\hline 4 & 303.8 & 22.8 & 306.0 & 39.1 & 307.1 & 63.9 \\
\hline 5 & 158.2 & 22.7 & 154.7 & 54.9 & 147.8 & 116.7 \\
\hline 6 & 185.4 & 7.1 & 184.6 & 16.2 & 183.9 & 33.3 \\
\hline 7 & 88.3 & 0.4 & 91.7 & 2.2 & 95.3 & 7.2 \\
\hline 8 & 192.1 & 15.9 & 195.4 & 39.7 & 202.4 & 86.4 \\
\hline 9 & 286.5 & 27.7 & 291.0 & 52.6 & 298.6 & 93.3 \\
\hline 10 & 261.3 & 19.0 & 258.1 & 33.6 & 254.4 & 57.8 \\
\hline 11 & 246.7 & 20.8 & 245.4 & 38.4 & 240.3 & 65.4 \\
\hline
\end{tabular}

TABLE 6: Link cost parameters for Example 2.

\begin{tabular}{lccc}
\hline Link & $t_{a}^{0}$ & $b_{a}$ & $c_{a}$ \\
\hline 1 & 1.2500 & 0.0253 & 1800 \\
2 & 1.2500 & 0.0253 & 1800 \\
3 & 9.1667 & 6.2610 & 1100 \\
4 & 9.1667 & 6.2610 & 1100 \\
5 & 9.1667 & 6.2610 & 1100 \\
6 & 2.5000 & 1.7075 & 1100 \\
7 & 7.5000 & 1.0866 & 1100 \\
8 & 9.1667 & 6.2610 & 1100 \\
9 & 2.5000 & 1.7075 & 1100 \\
10 & 7.5000 & 1.0866 & 1100 \\
11 & 2.5000 & 1.7075 & 1100 \\
12 & 2.5000 & 1.7075 & 1100 \\
13 & 2.0000 & 1.3660 & 1100 \\
14 & 7.5000 & 1.0866 & 1100 \\
15 & 7.5000 & 1.0866 & 1100 \\
16 & 2.0000 & 1.3660 & 1100 \\
17 & 1.2500 & 0.0253 & 1800 \\
18 & 1.2500 & 0.0253 & 1800 \\
\hline
\end{tabular}

TABLE 7: Mean of the log-normal demand for Example 2.

\begin{tabular}{lccc}
\hline Origin & \multicolumn{3}{c}{ Destination } \\
\hline 1 & 1 & 5 & 7 \\
5 & - & 600 & 400 \\
7 & 500 & - & 600 \\
\hline
\end{tabular}

assuming the demand function incorporates the uncertainty in an additive fashion. The other possible extensions are to also include the stochastic link capacity degradation in the analysis of the SN-MCP and to relax the strict assumption about constant VMR across all O-D pairs.
TABLE 8: Comparison between the true and estimated mean link flows and tolls under SN-SO.

\begin{tabular}{lcccc}
\hline & \multicolumn{2}{c}{ True results } & \multicolumn{2}{c}{ Estimated results } \\
Link & Mean flow & Toll & Mean flow & Toll \\
& $v_{a}$ & $\tau_{a}$ & $v_{a}$ & $\tau_{a}$ \\
\hline 1 & 875.0 & 0.01 & 887.0 & 0.01 \\
2 & 1000.0 & 0.02 & 1005.0 & 0.02 \\
3 & 437.5 & 3.31 & 448.4 & 3.35 \\
4 & 437.6 & 3.32 & 438.1 & 3.35 \\
5 & 500.0 & 4.72 & 495.9 & 4.73 \\
6 & 250.0 & 0.25 & 255.9 & 0.25 \\
7 & 187.5 & 0.09 & 189.4 & 0.09 \\
8 & 500.1 & 4.72 & 503.8 & 4.72 \\
9 & 250.1 & 0.25 & 252.9 & 0.25 \\
10 & 187.5 & 0.09 & 186.7 & 0.09 \\
11 & 326.1 & 0.44 & 325.6 & 0.45 \\
12 & 326.1 & 0.44 & 326.6 & 0.45 \\
13 & 800.0 & 4.05 & 792.0 & 4.09 \\
14 & 173.9 & 0.08 & 179.3 & 0.08 \\
15 & 174.0 & 0.08 & 175.3 & 0.07 \\
16 & 652.1 & 2.18 & 647.9 & 2.18 \\
17 & 1175.0 & 0.04 & 1172.0 & 0.04 \\
18 & 1000.0 & 0.02 & 994.5 & 0.02 \\
\hline
\end{tabular}

\section{Conflict of Interests}

The authors declare that there is no conflict of interests regarding the publication of this paper.

\section{Acknowledgments}

This research was supported by the Natural Science Foundation of China (NSFC) grants (Project nos. 71271112, 71201078, and 71371094) and the Research Grants Council (RGC) of Hong Kong (PolyU 5261/07E). 


\section{References}

[1] M. Z. F. Li, "Estimating congestion toll by using traffic count data-Singapore's area licensing scheme," Transportation Research Part E: Logistics and Transportation Review, vol. 35, no. 1, pp. 1-10, 1999.

[2] M. Z. F. Li, "The role of speed-flow relationship in congestion pricing implementation with an application to Singapore," Transportation Research Part B: Methodological, vol. 36, no. 8, pp. 731-754, 2002.

[3] W. Vickrey, "Point of view: principles and applications of congestion pricing," TR News, vol. 167, pp. 4-5, 1993.

[4] A. Downs, "Point of view: implementing peak-hour road pricing at fullscale: finding solutions to practical problems," $T R$ News, vol. 167, pp. 7-9, 1993.

[5] H. Yang, Q. Meng, and D.-H. Lee, "Trial-and-error implementation of marginal-cost pricing on networks in the absence of demand functions," Transportation Research Part B: Methodological, vol. 38, no. 6, pp. 477-493, 2004.

[6] H. Shao, W. H. Lam, and M. L. Tam, "A reliability-based stochastic traffic assignment model for network with multiple user classes under uncertainty in demand," Networks and Spatial Economics, vol. 6, no. 3-4, pp. 173-204, 2006.

[7] Z. Zhou and A. Chen, "Comparative analysis of three user equilibrium models under stochastic demand," Journal of Advanced Transportation, vol. 42, no. 3, pp. 239-263, 2008.

[8] B. W. Siu and H. K. Lo, "Doubly uncertain transportation network: degradable capacity and stochastic demand," European Journal of Operational Research, vol. 191, no. 1, pp. 166-181, 2008.

[9] G. R. Patil and S. V. Ukkusuri, "System-optimal stochastic transportation network design," Transportation Research Record, no. 2029, pp. 80-86, 2007.

[10] A. Chen and C. Yang, "Stochastic transportation network design problem with spatial equity constraint," Transportation Research Record, no. 1882, pp. 97-104, 2004.

[11] A. Sumalee, R. Connors, and D. Watling, "An optimal toll design problem with improved behavioural equilibrium model: the case of the probit model," in Mathematical and Computational Models for Congestion Charging, D. Hearn, S. Lawphongpanich, and M. Smith, Eds., pp. 219-240, Springer, Berlin, Germany, 2006.

[12] A. Sumalee, D. P. Watling, and S. Nakayama, "Reliable network design problem: case with uncertain demand and total travel time reliability," Transportation Research Record, no. 1964, pp. 81-90, 2006.

[13] A. Chen, J. Kim, Z. Zhou, and P. Chootinan, "Alpha reliable network design problem," Transportation Research Record, no. 2029, pp. 49-57, 2007.

[14] Y. Yin, S. M. Madanat, and X.-Y. Lu, "Robust improvement schemes for road networks under demand uncertainty," European Journal of Operational Research, vol. 198, no. 2, pp. 470479, 2009.

[15] A. Chen, Z. Zhou, P. Chootinan, S. Ryu, C. Yang, and S. C. Wong, "Transport network design problem under uncertainty: a review and new developments," Transport Reviews, vol. 31, no. 6, pp. 743-768, 2011

[16] S. D. Boyles, K. M. Kockelman, and S. Travis Waller, "Congestion pricing under operational, supply-side uncertainty," Transportation Research C: Emerging Technologies, vol. 18, no. 4, pp. 519-535, 2010.

[17] Z.-C. Li, W. H. K. Lam, S. C. Wong, and A. Sumalee, "Environmentally sustainable toll design for congested road networks with uncertain demand," International Journal of Sustainable Transportation, vol. 6, no. 3, pp. 127-155, 2012.

[18] S. Zhong, L. Zhang, and M. Bushell, "Reliability-based marginal cost pricing problem case with both demand uncertainty and travelers' perception errors," Mathematical Problems in Engineering, vol. 2013, Article ID 695307, 13 pages, 2013.

[19] N. Shirmohammadi, M. Zangui, Y. Yin, and Y. Nie, "Analysis and design of tradable credit schemes under uncertainty," Transportation Research Record, no. 2333, pp. 27-36, 2013.

[20] D. P. Watling, "Stochastic network equilibrium under stochastic demand," in Transportation Planning: State of the Art, M. Patriksson and M. Labbé, Eds., Kluwer, Dordrecht, The Netherlands, 2002.

[21] W. H. K. Lam, H. Shao, and A. Sumalee, "Modeling impacts of adverse weather conditions on a road network with uncertainties in demand and supply," Transportation Research B: Methodological, vol. 42, no. 10, pp. 890-910, 2008.

[22] M. G. H. Bell and C. Cassir, "Risk-averse user equilibrium traffic assignment: an application of game theory," Transportation Research Part B: Methodological, vol. 36, no. 8, pp. 671-681, 2002.

[23] M. Beckmann, C. McGuire, and C. Winsten, Studies in the Economics of Transportation, Yale University Press, New Haven, Conn, USA, 1956.

[24] A. Nagurney, Network Economics: A Variational Inequality Approach, Kluwer Academic Publishers, Norwell, Mass, USA, 2nd edition, 1999.

[25] Y. Zhao and K. M. Kockelman, "The propagation of uncertainty through travel demand models: an exploratory analysis," Annals of Regional Science, vol. 36, no. 1, pp. 145-163, 2002.

[26] N. Uno, F. Kurauchi, H. Tamura, and Y. Iida, "Using bus probe data for analysis of travel time variability," Journal of Intelligent Transportation Systems: Technology, Planning, and Operations, vol. 13, no. 1, pp. 2-15, 2009.

[27] L. F. Fenton, "The sum of log-normal probability distributions in scatter transmission systems," IEEE Transactions on Communication Systems, vol. 8, no. 1, pp. 57-67, 1960.

[28] W. B. Powell and Y. Sheffi, "The convergence of equilibrium algorithms with predetermined step sizes," Transportation Science, vol. 16, no. 1, pp. 45-55, 1982.

[29] S. T. Waller, J. L. Schofer, and A. K. Ziliaskopoulos, "Evaluation with traffic assignment under demand uncertainty," Transportation Research Record, no. 1771, pp. 69-74, 2001.

[30] A. Chen, K. Subprasom, and Z. Ji, "Mean-Variance model for the Build-Operate-Transfer scheme under demand uncertainty," Transportation Research Record, no. 1857, pp. 93-101, 2003. 


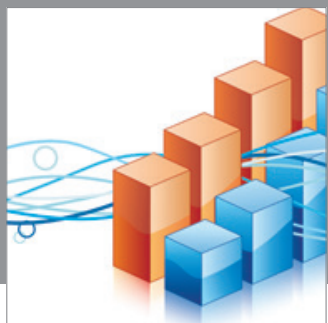

Advances in

Operations Research

mansans

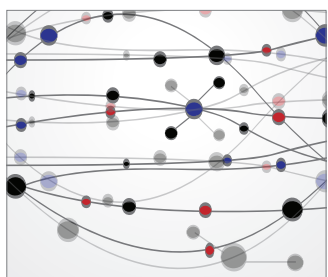

The Scientific World Journal
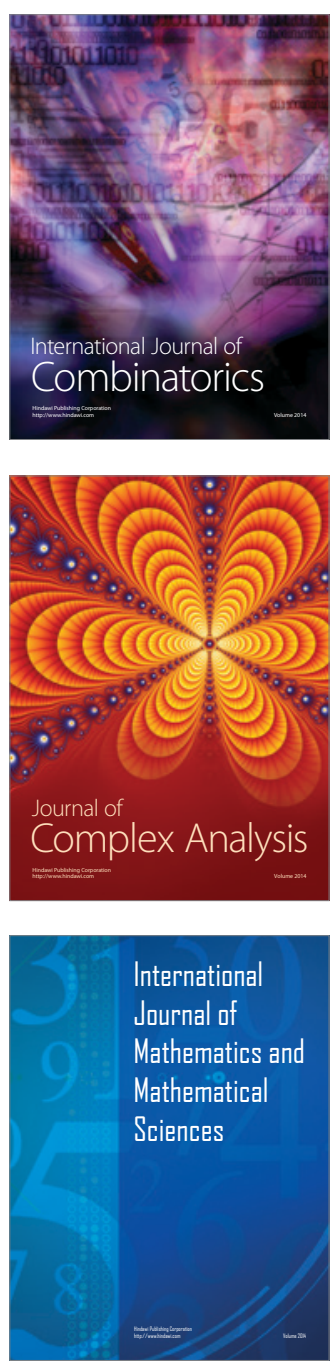
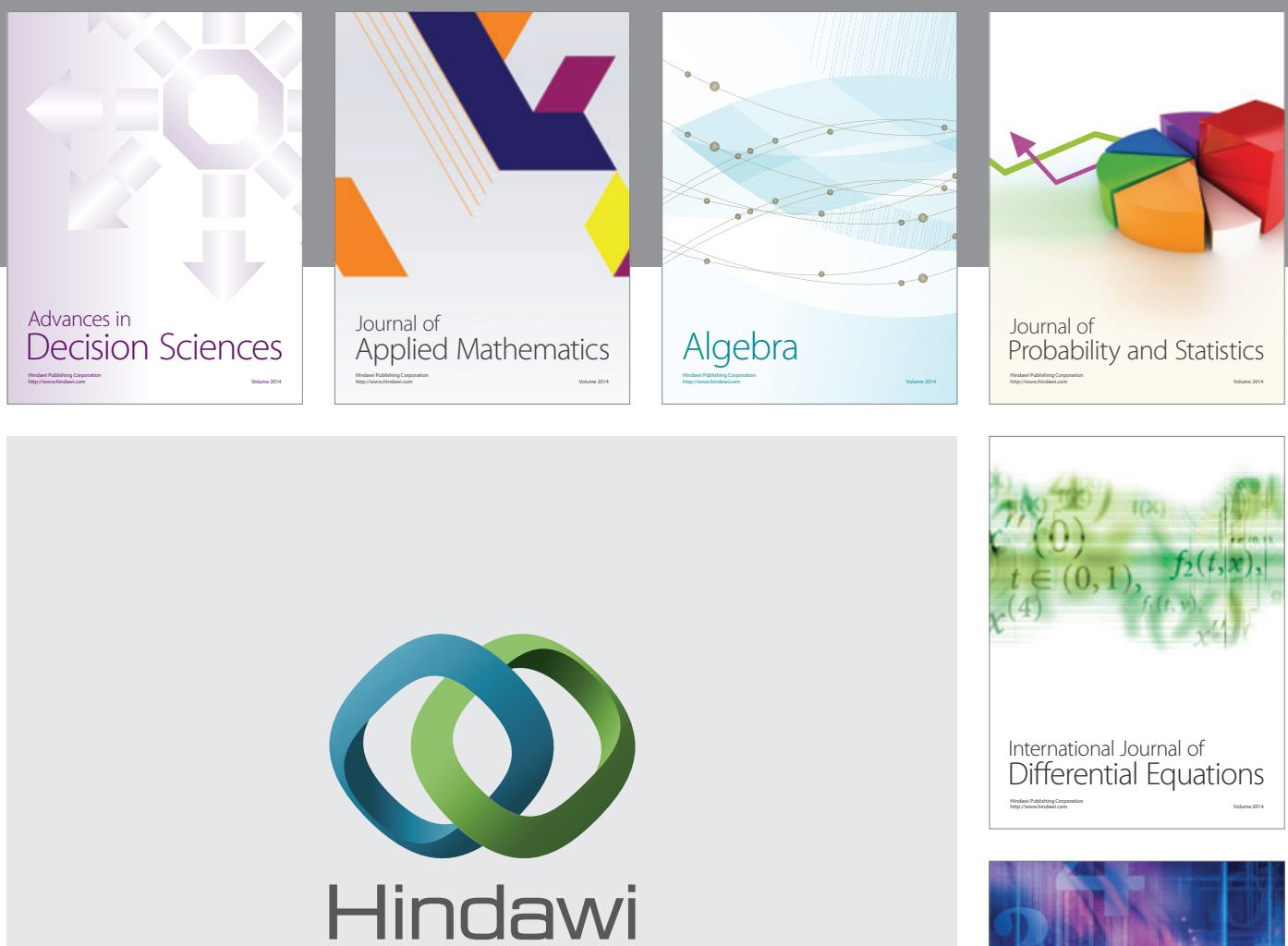

Submit your manuscripts at http://www.hindawi.com
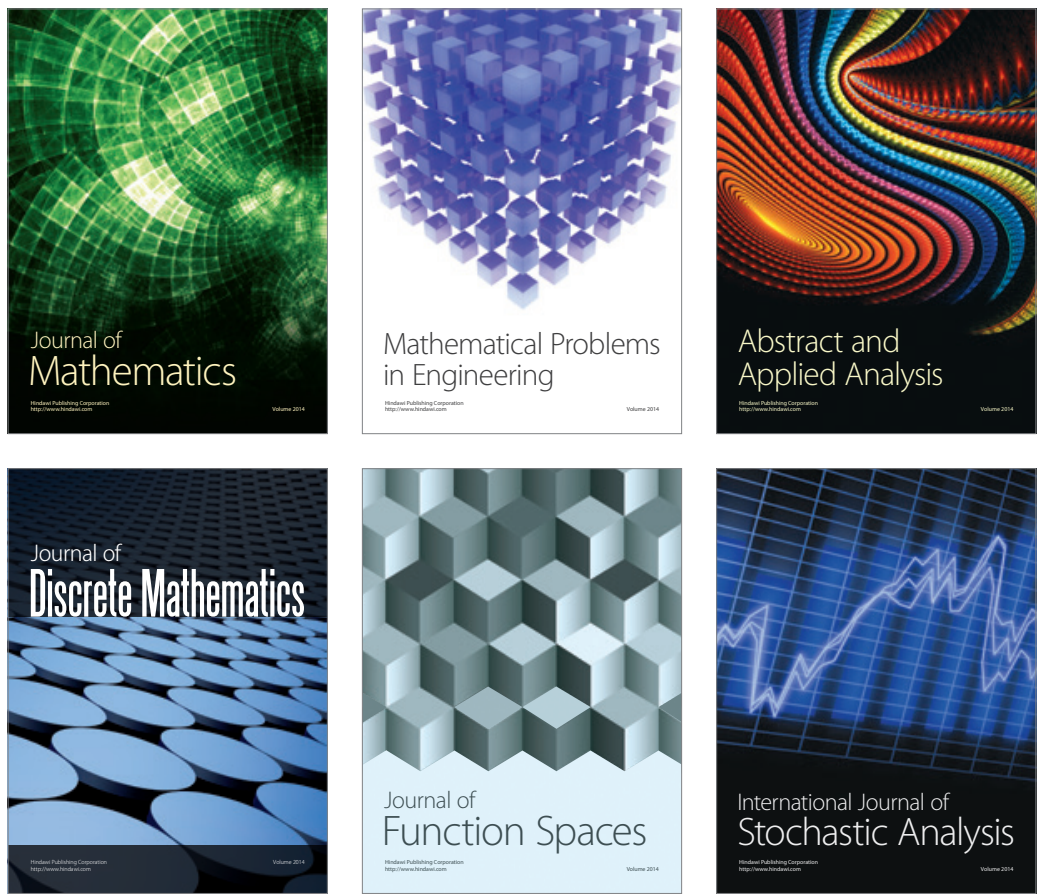

Journal of

Function Spaces

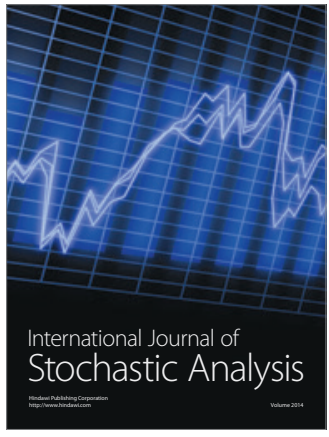

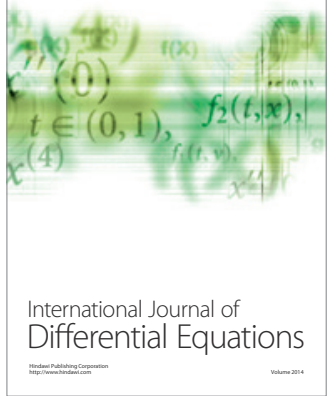
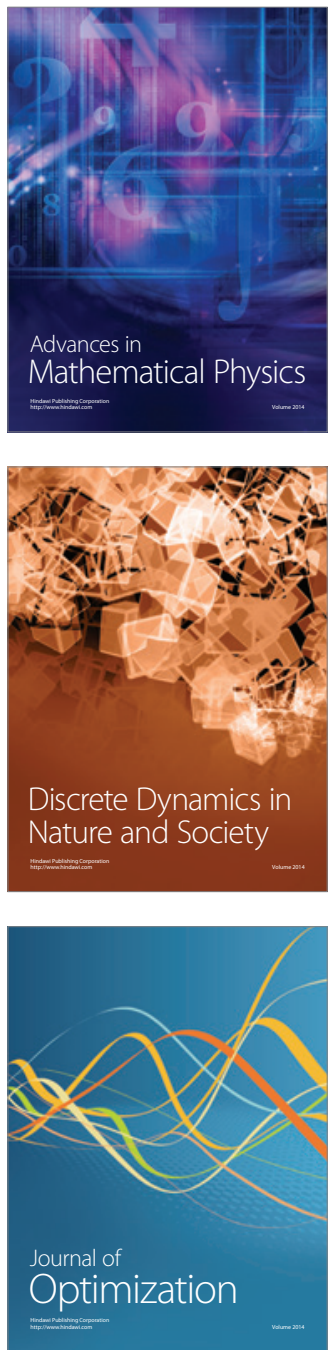